\title{
Treatment of Redundancies among Internal Coordinates in Optimizing Molecular Mechanics Force Constants
}

\author{
K. Palmö, ${ }^{*}$ L.-O. Pietilä, $\dagger$ and S. Krimm $\dagger \dagger$ \\ Biophysics Research Division and Department of Physics, University of Michigan, Ann Arbor, Michigan 48109
}

Received 15 October 1991; accepted 10 June 1992

\begin{abstract}
The use of redundant coordinate bases in the construction of molecular mechanics force fields is discussed. It is shown that the intrinsic indeterminacy in a force field in redundant coordinates in general stems from the squares of the first-order redundancy relations. The necessity to use constraints in such a force field is pointed out, and a method to check whether or not a set of constraints makes the force field determinate is described. It is also explained how force fields corresponding to different sets of constraints can be transformed into one another. To facilitate the utilization of $a b$ initio or other spectroscopic force fields, a procedure is given by which force constants pertaining to a nonredundant coordinate basis can be optimized in molecular mechanics calculations where redundant coordinates are used. (c) 1992 by John Wiley \& Sons, Inc.
\end{abstract}

\section{INTRODUCTION}

In the process of improving the accuracy of molecular mechanics calculations to account properly for vibrational frequencies, a larger number of interaction force constants than are necessary for the reproduction of correct structures has to be included in the potential energy functions. Because virtually all molecular mechanics programs use a set of internal coordinates that, for most molecules, contain many redundancies, there is then a danger of optimizing too many force constants independently. In such calculations, it is therefore important to take the redundancies correctly into account so that the potential energy parameters remain uniquely defined and intrinsic correlations between them are avoided. ${ }^{1}$ The utilization of $a b$ initio force fields in the construction of molecular mechanics energy functions also requires knowledge about the treatment of redundancies because the best way of implementing an $a b$ initio force field is to make a direct transformation of it into the molecular mechanics formalism, thus ensuring that subsequent simplifications and reoptimizations are on secure ground. ${ }^{2}$ In spectroscopic force field calculations, where the optimization of force fields to vibrational frequencies is the primary objective, nonredundant coordinates defined as linear combinations of the (redun-

\footnotetext{
${ }^{*}$ Current address: Department of Physics, Accelerator Laboratory, University of Helsinki, Siltavuorenpenger $20 \mathrm{M}, \mathrm{SF}-00170$ Helsinki, Finland.

†Current address: Neste Oy, Innopoli, P.O. Box 356, SF-02151 Espoo, Finland.

$\dagger \dagger$ Author to whom all correspondence should be addressed.
}

dant) basic internal coordinates are generally used, even if it sometimes would be more convenient to use directly the redundant set of coordinates. However, in performing molecular mechanics calculations, where minimization of the potential energy or other operations in the conformational space (such as molecular dynamics) are the main interest, there has so far been no need to implement nonredundant coordinates. In fact, explicit inclusion of all the basic internal coordinates makes it easier to evaluate the computed structures. In these calculations, the redundancies are also effectively eliminated by the use of Cartesian coordinates in the actual computations. On the other hand, when constructing a force field in redundant coordinates the situation is different, and careless optimization of a large number of force constants may produce physically meaningless energy parameters, although the frequency fit may be good. Similar effects can of course also be caused by correlations due to an insufficient number of data in the fit, but this can be corrected by using a larger data set. However, indeterminacy caused by redundancies remains present regardless of the number of data.

There are basically two types of redundancies: local and cyclic. Local redundancies occur in trigonal and tetrahedral configurations if every valence angle is chosen as a separate coordinate and in torsions if several torsion coordinates are defined for a bond. Cyclic redundancies occur in ring structures and may involve all types of internal coordinates, often in a quite complicated manner. Benzene, cyclopentane, and cyclobutane are well-known molecules that have cyclic redundancies among their valence coordinates. A molecular mechanics energy function 
also contains nonbonded atom-atom interactions that, together with the valence force field, describe the potential energy surface of the molecule in question. The nonbonded interactions introduce, in principle, still more redundancies in the energy function because many closed rings are formed (cf. UreyBradley force field), but usually this is not a problem because the parameters of the nonbonded interactions are determined independently of the force constants.

Many articles dealing with redundancies among internal coordinates in spectroscopic force field calculations have previously been published. ${ }^{3-10}$ However, in molecular mechanics calculations the fact that the actual geometry generally differs from the reference geometry, with respect to which the force field is defined, makes a new analysis necessary. ${ }^{1}$ In this article, we explain the origin of the redundancies in a new way and show why they cannot be ignored. We also describe a method by which they can be taken into account in molecular mechanics calculations, while still retaining the convenient redundant coordinate basis, as well as the automatic uniqueness and better transferability of parameters associated with a nonredundant set of coordinates.

\section{INDETERMINACY CAUSED BY REDUNDANCIES}

In the following, we assume that the parameters of the nonbonded interactions are optimized independently of the force constants so that the nonbonded interactions merely deform the potential energy surface defined by the intrinsic valence force field without causing any correlations between the energy parameters.

The potential energy pertaining to the deformation of the internal coordinates of a molecule is usually of the form

$$
V=\frac{1}{2} \Delta \mathbf{q}^{t} \mathbf{F} \Delta \mathbf{q}
$$

where $\mathbf{F}$ is the force constant matrix and the vector $\Delta \mathbf{q}=\mathbf{q}-\mathbf{q}_{0}$ describes the deformation of the internal coordinates, contained in the vector $\mathbf{q}$, from their intrinsic equilibrium values represented by the vector $\mathbf{q}_{0}$. If the internal coordinates are not linearly independent but contain redundancies, the dimension of the conformational space is smaller than the number of internal coordinates. The conformational space is then a subspace (hypersurface) in the full internal coordinate space. Obviously, the molecular geometry can never represent a point outside this hypersurface. Figure 1 illustrates (in three dimensions) the general case of a curved conformational surface in a redundant coordinate space. A simple example of a real conformational surface is given by the relation between the three valence angles in a

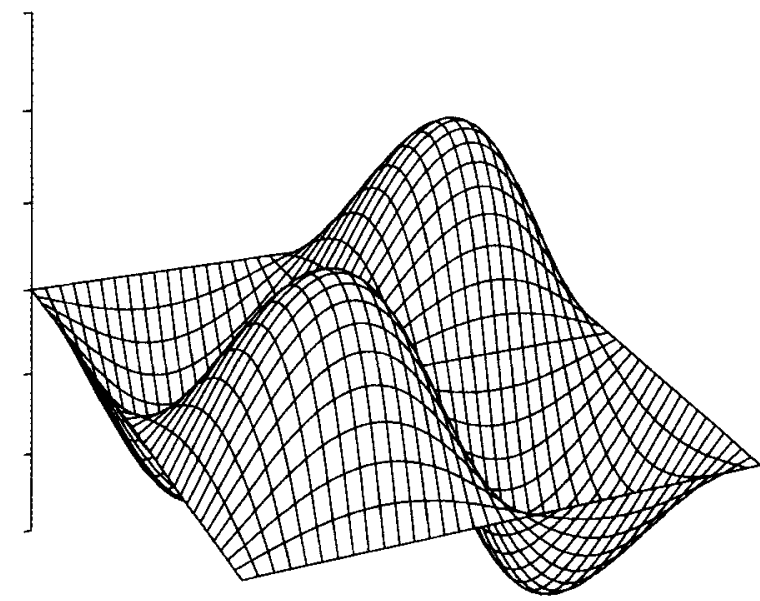

Figure 1. Two-dimensional conformational space (hypersurface) in a three-dimensional redundant internal coordinate space. The three mutually perpendicular axes represent the three coordinates that mathematically can be considered independent but are interrelated by the geometric properties of the molecule. The relation between the coordinates is a function that makes up the curved surface, the conformational surface. Note that this does not illustrate a potential energy surface, the energy being a fourth variable that depends upon the molecular coordinates located on the surface.

planar trigonal system (e.g., around an $s p^{2}$ carbon atom). The sum of the three angles is always $360^{\circ}$ (provided that the configuration stays planar), and the conformational space is therefore a triangular plane formed by connecting the points $\left(360^{\circ}, 0,0\right)$, $\left(0,360^{\circ}, 0\right)$, and $\left(0,0,360^{\circ}\right)$. If there are $N_{r}$ redundancies among the internal coordinates, there exist $N_{r}$ linearly independent functions, $\phi_{r}(\mathbf{q}), r=$ $1, \ldots, N_{r}$, that are zero for every vector $\mathbf{q}$ that represents a point on the conformational hypersurface. Writing the redundancy functions $\phi_{r}(\mathbf{q})$ as Taylor series, we have

$$
\phi_{r}(\mathbf{q})=\mathbf{a}_{r}^{l} \Delta \tilde{\mathbf{q}}_{r}+\frac{1}{2} \Delta \tilde{\mathbf{q}}_{r}^{l} \mathbf{A}_{r} \Delta \tilde{\mathbf{q}}_{r}+\ldots=0
$$

where $r=1, \ldots, N_{r}$. Here, $\Delta \tilde{\mathbf{q}}_{r}=\mathbf{q}-\mathbf{q}_{e x, r}$ denotes the displacement of the molecular geometry from some expansion center $\mathbf{q}_{e x, r}$ in the conformational space, and the vector $\mathbf{a}_{r}$ and the matrix $\mathbf{A}_{r}$ contain the first- and second-order expansion coefficients, respectively. It is worth noting that the vector $\mathbf{q}_{0}$ does not necessarily satisfy eq. (2). Cyclobutane, for example, is a drastic case where the intrinsic equilibrium values of the $\mathrm{C}-\mathrm{C}-\mathrm{C}$ valence angles do not satisfy the cyclic redundancy relation. (Clearly, the four $\mathrm{C}-\mathrm{C}-\mathrm{C}$ angles cannot all simultaneously be, say, $113.5^{\circ}$ ). The expansion center $\mathbf{q}_{e x, r}$ is arbitrary, but one of the objectives of the following discussion is to determine the locations of $\mathbf{q}_{e x, r}$ for which indeterminacy may arise in the force field. It is useful to express $\Delta \tilde{\mathbf{q}}_{r}$ in terms of $\Delta \mathbf{q}$, which yields

$$
\Delta \tilde{\mathbf{q}}_{r}=\Delta \mathbf{q}-\left(\mathbf{q}_{e x, r}-\mathbf{q}_{0}\right)=\Delta \mathbf{q}-\Delta \mathbf{q}_{e x, r}
$$


where we have defined

$$
\Delta \mathbf{q}_{e x, r}=\mathbf{q}_{e x, r}-\mathbf{q}_{0}
$$

The indeterminacy in the energy parameters comes about because terms that depend upon the redundancy functions $\phi_{r}(\mathbf{q})$ can be added to the en ergy function without changing the potential energy surface in any way. Thus

$$
V_{1}=V+\sum_{r}\left[\kappa_{r} \phi_{r}(\mathbf{q})+\frac{1}{2} \gamma_{r} \phi_{r}^{2}(\mathbf{q})+\ldots\right]
$$

(where $\kappa_{r}, \gamma_{r}$, etc. are constants) represents exactly the same potential energy as $V$. The force constant matrix is then not necessarily uniquely defined if there exist redundant energy terms that can be written in the same form (1) as the energy spanned by the force constants. To investigate this further, let us look closer at the quadratic term in eq. (4). For the moment, we assume that $\mathbf{q}$ is not far from $\mathbf{q}_{e x, r}$ so that terms of third and higher order with respect to $\Delta \tilde{\mathbf{q}}_{r}$ can be neglected. Let us also assume that $\mathbf{q}_{0}$, if it belongs to the conformational space, is correspondingly close to $\mathbf{q}_{e x, r}$. We then have

$$
\begin{aligned}
\frac{1}{2} \gamma_{r} \phi_{r}^{2}(\mathbf{q})= & \frac{1}{2} \gamma_{r}\left(\Delta \tilde{\mathbf{q}}_{r}^{t} \mathbf{a}_{r}+\frac{1}{2} \Delta \tilde{\mathbf{q}}_{r}^{t} \mathbf{A}_{r} \Delta \tilde{\mathbf{q}}_{r}\right) \\
& \times\left(\mathbf{a}_{r}^{t} \Delta \tilde{\mathbf{q}}_{r}+\frac{1}{2} \Delta \tilde{\mathbf{q}}_{r}^{t} \mathbf{A}_{r} \Delta \tilde{\mathbf{q}}_{r}\right) \\
= & \frac{1}{2} \gamma_{r}\left(\Delta \tilde{\mathbf{q}}_{r}^{t} \mathbf{a}_{r} \mathbf{a}_{r}^{t} \Delta \tilde{\mathbf{q}}_{r}\right)=0
\end{aligned}
$$

Substitution of eq. (2) and (5) into eq. (4) then yields (to the second order)

$$
\begin{aligned}
& V_{\mathrm{l}}=V+ \\
& \sum_{r}\left[\kappa_{r} \mathbf{a}_{r}^{t} \Delta \tilde{\mathbf{q}}_{r}+\frac{1}{2} \Delta \tilde{\mathbf{q}}_{r}^{t}\left(\kappa_{r} \mathbf{A}_{r}+\gamma_{r} \mathbf{a}_{r} \mathbf{a}_{r}^{t}\right) \Delta \tilde{\mathbf{q}}_{r}\right]
\end{aligned}
$$

Using eq. (3a) to obtain explicit dependence upon $\Delta \mathbf{q}$, we then have

$$
\begin{aligned}
V_{1}=\sum_{r} & {\left[\frac{1}{2} \Delta \mathbf{q}_{e x, r}^{t}\left(\kappa_{r} \mathbf{A}_{r}+\gamma_{r} \mathbf{a}_{r} \mathbf{a}_{r}^{t}\right) \Delta \mathbf{q}_{e x, r}\right.} \\
& \left.-\kappa_{r} \mathbf{a}_{r}^{t} \Delta \mathbf{q}_{e x, r}\right] \\
& -\sum_{r}\left[\Delta \mathbf{q}_{e x, r}^{t}\left(\kappa_{r} \mathbf{A}_{r}+\gamma_{r} \mathbf{a}_{r} \mathbf{a}_{r}^{t}\right)-\kappa_{r} \mathbf{a}_{r}^{t}\right] \Delta \mathbf{q} \\
& +\frac{1}{2} \Delta \mathbf{q}^{t}\left[\mathbf{F}+\sum_{r}\left(\kappa_{r} \mathbf{A}_{r}+\gamma_{r} \mathbf{a}_{r} \mathbf{a}_{r}^{t}\right)\right] \Delta \mathbf{q}
\end{aligned}
$$

The force constant matrix is indeterminate if some of its elements can be continuously modified in a way that does not affect the potential energy. Equation (7) suggests that the force constants can change in any way that is equivalent to addition of the sum

$$
\sum_{r}\left(\kappa_{r} \mathbf{A}_{r}+\gamma_{r} \mathbf{a}_{r} \mathbf{a}_{r}^{t}\right)
$$

to the $\mathbf{F}$ matrix. Clearly, the $\mathbf{F}$ matrix is not well defined if an exprfession like (8), containing free parameters, can be added to it without causing any change in the potential energy. However, although eq. (7) always represents the same potential energy surface as eq. (1), all of the potential energy represented by eq. (7) is not necessarily contained in the second-order terms [last row of eq. (7)]. Obviously, no indeterminacy results from the addition of such quadratic redundant terms that need to be compensated by linear or constant terms to preserve the potential energy. This is because such a change in the force field in reality changes the potential energy because linear or constant terms are not included in the molecular mechanics energy function. Thus, the redundancies make the force constant matrix indeterminate only if the potential energy spanned by the sum (8) vanishes, and because the redundancy relations are linearly independent we must then have

$$
\Delta \mathbf{q}^{l}\left(\kappa_{r} \mathbf{A}_{r}+\gamma_{r} \mathbf{a}_{r} \mathbf{a}_{r}^{l}\right) \Delta \mathbf{q}=0
$$

for every $r=1, \ldots, N_{r}$. As we shall see, this condition alone fixes the constants $\kappa_{r}$ to zero and thus removes the indeterminacy caused by the redundancy matrices $\mathbf{A}_{r}$. To show this, we assume that the redundancy relation under consideration is nonlinear, i.e., that $\mathbf{A}_{r}$ is not the zero matrix. Substituting $\Delta \mathbf{q}=$ $\Delta \tilde{\mathbf{q}}_{r}+\Delta \mathbf{q}_{e x, r}$ into eq. (9), and noting that by eq. (2) $\Delta \tilde{\mathbf{q}}_{r}^{t} \mathbf{A}_{r} \Delta \tilde{\mathbf{q}}_{r}=-2 \mathbf{a}_{r}^{t} \Delta \tilde{\mathbf{q}}_{r}$ and that by eq. (5) $\Delta \tilde{\mathbf{q}}_{r}^{t}\left(\gamma_{r} \mathbf{a}_{r} \mathbf{a}_{r}^{t}\right) \Delta \tilde{\mathbf{q}}_{r}=0$, we get

$$
\begin{aligned}
& -2 \kappa_{r} \mathbf{a}_{r}^{t} \Delta \tilde{\mathbf{q}}_{r}+2 \kappa_{r} \Delta \mathbf{q}_{e x, r}^{t} \mathbf{A}_{r} \Delta \tilde{\mathbf{q}}_{r}+\kappa_{r} \Delta \mathbf{q}_{e x, r}^{t} \mathbf{A}_{r} \Delta \mathbf{q}_{e x, r} \\
& \quad+2 \Delta \mathbf{q}_{e x, r}^{t}\left(\gamma_{r} \mathbf{a}_{r} \mathbf{a}_{r}^{l}\right) \Delta \tilde{\mathbf{q}}_{r}+\Delta \mathbf{q}_{e x, \mathrm{r}}^{t}\left(\gamma_{r} \mathbf{a}_{r} \mathbf{a}_{r}^{t}\right) \Delta \mathbf{q}_{e x, r}=0
\end{aligned}
$$

Here, it may be argued that if the force constants are optimized only to vibrational frequencies and geometry data there is still indeterminacy in the $\mathbf{F}$ matrix if eqs. (9) and (10) yield any constant, not necessarily zero. Therefore, we also have to keep in mind the possibility that only the linear terms of eq. (10) vanish. The question is then: For what values of $\mathbf{q}_{e x, r}$ does eq. (10) yield zero, or a constant, in a way that leaves freedom to the parameters $\kappa_{r}$ and $\gamma_{r}$. This determines the nature of the indeterminacy. When optimizing a force field, this indeterminacy has to be removed externally by imposing constraints on the force constants.

As previously mentioned, the vector $q_{0}$, which denotes the intrinsic equilibrium geometry, may or may not satisfy the redundancy relations. Let us first assume that $\mathbf{q}_{0}$ does satisfy all the redundancy relations. In this case, we may have $\mathbf{q}_{e x, r}=\mathbf{q}_{0}$ and eq. (10) becomes

$$
-2 \kappa_{r} \mathbf{a}_{r}^{t} \Delta \tilde{\mathbf{q}}_{r}=0
$$

which means that $\kappa_{r}$ must be zero because we assume that the redundancy relation is nonlinear. Obviously, eq. (11) cannot yield a constant other than 
zero. Having $\mathbf{q}_{e x, r}=\mathbf{q}_{0}$ thus fixes $\boldsymbol{\kappa}_{r}$ to zero but leaves total freedom to $\gamma_{r}$. Let us see whether there are any other values of $\mathbf{q}_{e x, r}$ that allow freedom to $\kappa_{r}$ and/or $\gamma_{r}$. If $\mathbf{q}_{e x, r} \neq \mathbf{q}_{0}$ one constant term and three linear terms remain in eq. (10). The linear terms cannot yield a nonzero constant but must vanish for every vector $\Delta \tilde{\mathbf{q}}_{r}$ in the conformational space. Thus, we must have

$$
\begin{aligned}
-\kappa_{r} \mathbf{a}_{r}^{t} \Delta \tilde{\mathbf{q}}_{r}+\kappa_{r} \Delta \mathbf{q}_{e x, r}^{t} \mathbf{A}_{r} \Delta \tilde{\mathbf{q}}_{r} & +\Delta \mathbf{q}_{e x, r}^{t}\left(\gamma_{r} \mathbf{a}_{r} \mathbf{a}_{r}^{t}\right) \Delta \tilde{\mathbf{q}}_{r}=0
\end{aligned}
$$

This must also be true for $\mathbf{q}=\mathbf{q}_{0}$, which gives

$$
\begin{aligned}
\kappa_{r} \mathbf{a}_{r}^{i} \Delta \mathbf{q}_{e x, r}-\kappa_{r} \Delta \mathbf{q}_{e x, r}^{t} \mathbf{A}_{r} \Delta \mathbf{q}_{e x, r} \\
-\Delta \mathbf{q}_{e x, r}^{t}\left(\gamma_{r} \mathbf{a}_{r} \mathbf{a}_{r}^{t}\right) \Delta \mathbf{q}_{e x, r}=0
\end{aligned}
$$

Here, the last term vanishes by eq. (5) and if we use eq. (2) with $\mathbf{q}=\mathbf{q}_{0}$ eq. (13) reduces to

$$
\kappa_{r} \mathbf{a}_{r}^{t} \Delta \mathbf{q}_{e x, r}=0
$$

which cannot hold true unless $\kappa_{r}=0$. Substitution of $\kappa_{r}=0$ back into eq. (10) then yields

$$
2 \Delta \mathbf{q}_{e x, r}^{t}\left(\gamma_{r} \mathbf{a}_{r} \mathbf{a}_{r}^{i}\right) \Delta \tilde{\mathbf{q}}_{r}=0
$$

the left-hand side of which is linear with respect to $\Delta \tilde{\mathbf{q}}_{r}$ and is not zero to the second order unelss $\gamma_{r}=$ 0 (or $\mathbf{q}_{e x, r}=\mathbf{q}_{0}$, or the redundancy relation is linear). Thus, for a nonlinear redundancy relation there is no freedom for $\boldsymbol{\kappa}_{r}$, nor for $\gamma_{r}$, if $\mathbf{q}_{e x, r} \neq \mathbf{q}_{0}$. Evidently, if the redundancy relation is linear the vector $\mathbf{a}_{r}$ does not depend upon the location of the expansion center and eqs. (9) and (10) then leave full freedom to $\gamma_{r}$ for any location of $\mathbf{q}_{e x, r}$.

We still have to consider the case that $\mathbf{q}_{0}$ does not satisfy one or more of the redundancy relations, i.e., that eq. (2) is not always true for $\mathbf{q}=\mathbf{q}_{0}$. This then means that $\mathbf{q}_{0}$ is located outside the conformational surface, a situation that may occur, for instance, in strained ring structures. In this case, of course, $\mathbf{q}_{e x, r}$ cannot be equal to $\mathbf{q}_{0}$ because $\mathbf{q}_{e x, r}$ always represents a point in the conformational space. However, even if $\mathbf{q}_{0}$ does not satisfy the $r$ th redundancy relation eq. (12) must still hold if the potential energy is not to be changed by the addition of (8) to the force constant matrix. Regrouping the terms in eq. (12), we have

$$
\kappa_{r} \Delta \mathbf{q}_{e x, r}^{t} \mathbf{A}_{r} \Delta \tilde{\mathbf{q}}_{r}+\left(\gamma_{r} \Delta \mathbf{q}_{e x, r}^{t} \mathbf{a}_{r}-\boldsymbol{\kappa}_{r}\right) \mathbf{a}_{r}^{t} \Delta \tilde{\mathbf{q}}_{r}=0
$$

Then, the expansion center $\mathbf{q}_{e x, r}$ must be such that

$$
\mathbf{a}_{r}^{t} \Delta \tilde{\mathbf{q}}_{r}=\alpha_{r} \Delta \mathbf{q}_{e x, r}^{t} \mathbf{A}_{r} \Delta \tilde{\mathbf{q}}_{r}
$$

where

$$
\alpha_{r}=\frac{\boldsymbol{\kappa}_{r}}{\boldsymbol{\kappa}_{r}-\gamma_{r} \Delta \mathbf{q}_{e x, r}^{t} \mathbf{a}_{r}}
$$

with the provision that

$$
\kappa_{r} \neq \gamma_{r} \Delta \mathbf{q}_{e x, r}^{t} \mathbf{a}_{r}
$$

Substituting $\mathbf{a}_{r}^{t} \Delta \tilde{\mathbf{q}}_{r}$ of eq. (17) into the redundancy relation (2), we get

$$
\left(\alpha_{r} \Delta \mathbf{q}_{e x, r}^{l}+\frac{1}{2} \Delta \tilde{\mathbf{q}}_{r}^{t}\right) \mathbf{A}_{r} \Delta \tilde{\mathbf{q}}_{r}=0
$$

Obviously, $\Delta \overline{\mathbf{q}}_{r}=-2 \alpha_{r} \Delta \mathbf{q}_{e x, r}$ satisfies eq. (18). However, this vector $\Delta \tilde{\mathbf{q}}_{r}$ cannot satisfy the original redundancy relation because it has a component outside the conformational space for every nonzero value of $\alpha_{r}$. If $\alpha_{r}=0$, eq. (17) gives $\mathbf{a}_{r} \Delta \tilde{\mathbf{q}}_{r}=0$, which is not true if the redundancy relation is nonlinear. Thus, eq. (18) is not identical to eq. (2) and hence an expansion center $\Delta \mathbf{q}_{e x, r}$ that satisfies eq. (17) cannot exist. We now go back to eq. (16) and consider the case that $\kappa_{r}=\gamma_{r} \Delta \mathbf{q}_{e x, r}^{t} \mathbf{a}_{r}$, which was excluded in eq. (17). Equation (16) then gives

$$
\kappa_{r} \Delta \mathbf{q}_{e x, r}^{t} \mathbf{A}_{r} \Delta \tilde{\mathbf{q}}_{r}=0
$$

If the redundancy relation is nonlinear, $\Delta \mathbf{q}_{e x, r}^{l} \mathbf{A}_{r}$ (or any other constant vector) cannot be orthogonal to every vector $\Delta \tilde{\mathbf{q}}_{r}=0$ in the conformational space, so if $\kappa_{r} \neq 0$ we must have

$$
\Delta \mathbf{q}_{e x, r}^{t} \mathbf{A}_{r}=\mathbf{O}^{t}
$$

This would mean that there exists an expansion center $\mathbf{q}_{e x, r}$ for which the $\mathbf{A}_{r}$ matrix has at least one eigenvalue that is zero, and that $\Delta \mathbf{q}_{e x, r}$ is the corresponding eigenvector. The $\mathbf{A}_{r}$ matrix has a zero eigenvalue if the redundancy relation is linear in some direction, in a neighborhood of $\mathbf{q}_{e x, r}$. But, $\Delta \mathbf{q}_{e x, r}=\mathbf{q}_{e x, r}-\mathbf{q}_{0}$ cannot be the eigenvector of such a matrix $\mathbf{A}_{r}$ because this would require $\mathbf{q}_{0}$ to be located in the linear region of the subspace defined by the redundancy relation, and $\mathbf{q}_{0}$ would then satisfy the redundancy relation, in contradiction with our original assumption. Thus, eq. (16) cannot be true unless $\kappa_{r}=0$. Substituting $\kappa_{r}=0$ into eq. (16) gives

$$
\gamma_{r}\left(\Delta \mathbf{q}_{e x, r}^{t} \mathbf{a}_{r}\right) \mathbf{a}_{r}^{t} \Delta \overline{\mathbf{q}}_{r}=0
$$

This is zero to the second order for all values of $\gamma_{r}$ if $\Delta \mathbf{q}_{e x, r}^{l} \mathbf{a}_{r}=0$ or the redundancy relation is linear. We cannot rule out the possibility of the existence of an expansion center $\mathbf{q}_{e x, r}$ that makes $\Delta \mathbf{q}_{e x, r}^{t} \mathbf{a}_{r}=0$ for a nonlinear redundancy relation. Such a $\mathbf{q}_{e x, r}$ exists if the hypersurface defined by the redundancy relation has a tangent that goes through $\mathbf{q}_{0}$. If we look at the second row of eq. (10), we can see that in this case the last (constant) term also disappears and we have a true indeterminacy. However, if the redundancy relation is linear no tangent to the hypersurface can go through $\mathbf{q}_{0}$, and the addition of $\gamma_{r} \mathbf{a}_{r} \mathbf{a}_{r}^{t}$ to the force constant matrix is then equivalent to the addition of a constant to the potential energy. The constant term depends upon $\gamma_{r}$ and $\mathbf{q}_{0}$ but not upon the choice of $\mathbf{q}_{e x, r}$. The addition of a constant to the potential energy does not, or course, affect the molecular geometry or the vibrational frequencies, so from a practical point of view the force con- 
stant matrix is still indeterminate if no energy data are used in the optimization.

The preceding results were derived under the assumption that $\mathbf{q}, \mathbf{q}_{0}$, and $\mathbf{q}_{e x, r}$ were close to one another so that a second-order expansion of each redundancy function was sufficient. We proved that a redundancy relation, satisfied by $\mathbf{q}_{0}$, causes indeterminacy in the force constant matrix only when the expansion center $\mathbf{q}_{e x, r}=\mathbf{q}_{0}$, that is, $\Delta \tilde{\mathbf{q}}_{r}=\Delta \mathbf{q}$. However, from eqs. (1) and (6) it is then obvious that terms of third and higher order with respect to $\Delta \mathbf{q}$ could only affect energy parameters related to such higher terms. Further, because the second-order expansion matrices $\mathbf{A}_{r}$ were shown not to cause any indeterminacy (because the $\kappa_{r}$ must be zero), the only term in (8) and (9) that needs to be considered is $\gamma \mathbf{a}_{r} \mathbf{a}_{r}^{t}$. Thus, as is seen from eq. (5), the indeterminacy then stems entirely from the squares of the first-order redundancy relations. For a nonlinear redundancy relation that $\mathbf{q}_{0}$ does not satisfy, there is, however, a chance that higher-order terms may come into effect. This happens if the values of $\mathbf{q}_{e x, r}$ for which eq. (21) is valid (if any such values exist) are so far away from $\mathbf{q}$ that a second-order expansion of the redundancy relation is not sufficient. In this case, the nature of the indeterminacy is not fully described by the analysis made here.

We may now draw some conclusions about the effect of a redundancy on the force constant matrix. If $\mathbf{q}_{0}$ belongs to the conformational space and $\mathbf{q}$ is close enough to $\mathbf{q}_{0}$ for the redundancy relation to be considered linear, the factors $\Delta \mathbf{q}^{t} \mathbf{a}_{r}$ and $\mathbf{a}_{r}^{t} \Delta \mathbf{q}$ in eq. (9) are both zero. Either $\mathbf{a}_{r}$ or $\mathbf{a}_{r}^{t}$ can then be replaced by an arbitrary vector (of the same dimension) $h_{r}$ or $h_{r}^{l}$, respectively, and eq. (9) will still yield zero. In this case, we can therefore replace the symmetric matrix $\mathbf{a}_{r} \mathbf{a}_{r}^{t}$ with the more general symmetric matrix $\mathbf{a}_{r} \mathbf{h}_{r}^{t}+\mathbf{h}_{r} \mathbf{a}_{r}^{t}$. The parameter $\gamma_{r}$ may then be considered to be included in the vector $\mathbf{h}_{r}$, and the matrix that can be added to the force constant matrix without changing the potential energy is

$$
\tilde{\mathbf{F}}_{r}=\mathbf{a}_{r} \mathbf{h}_{r}^{t}+\mathbf{h}_{r} \mathbf{a}_{r}^{t}
$$

where the vector $\mathbf{a}_{r}$ is calculated at $\mathbf{q}_{e x, r}=\mathbf{q}_{0}$. This represents maximum indeterminacy, i.e., the largest possible number of degrees of freedom for the $\tilde{\mathbf{F}}_{r}$ matrix. Note that the number of constraints needed to eliminate this indeterminacy is equal to or larger than the number of internal coordinates. If $\mathbf{q}$ is so far away from $\mathbf{q}_{0}$ that the redundancy relation can no longer be considered linear, the indeterminacy is in principle less serious, and instead of eq. (22a) we then have

$$
\tilde{\mathbf{F}}_{r}=\gamma_{r} \mathbf{a}_{r} \mathbf{a}_{r}^{t}
$$

which contains only one free parameter. Theoretically, the indeterminacy of the force constants even disappears for sufficiently large $\Delta \mathbf{q s}$ if the secondorder Taylor series fails to describe the redundancy relation properly. This is because the addition of $\tilde{\mathbf{F}}_{r}$ to the force constant matrix then changes the potential energy. However, it seems difficult to utilize this, or eq. (22b), for any practical purposes because the indeterminacy given by eq. (22a) decreases only in accordance with the nonlinearity of the redundancy relation, and hence the potential energy and the vibrational frequencies are not sensitive to the force constants in the same way as if the redundancy would not exist. A well-taken precaution is therefore to assume that the indeterminacy is given by eq. (22a). This also holds even if $\mathbf{q}_{0}$ only approximately satisfies the redundancy relation.

If $\mathbf{q}_{0}$ does not even approximately belong to the conformational space, we have the following possibilities: If the redundancy relation is linear, the indeterminacy in the force field is of type (22b). Strictly speaking, however, the potential energy is then not indeterminate because the addition of eq. (22b) to the $\mathbf{F}$ matrix causes the energy surface to move up or down, even though its form does not change. If the redundancy relation is nonlinear, but all values of $\mathbf{q}_{e x, r}$ (if any) for which eq. (21) holds are close enough to $\mathbf{q}$ to make the second-order Taylor series valid, the indeterminacy in the $\mathbf{F}$ matrix is still of type (22b) but now the potential energy itself is also indeterminate. If the second-order expansion is not sufficient, the nature of the indeterminacy may be more complicated. However, it is not worthwhile trying to deduce the nature of the indeterminacy in this case because when optimizing a molecular mechanics force field involving molecules with such redundancies other molecules where the problematic redundancies do not occur are usually also included in the calculation and the indeterminacy is thereby removed. Alternatively, if one always assumes that the indeterminacy is of type (22a) the constraints then applied are likely also to take care of possible indeterminacy not adequately described by a second-order expansion of the redundancy function.

\section{ELIMINATION OF THE INDETERMINACY}

In the previous section, it was shown that, to eliminate the indeterminacy the redundancies cause in the force field, it is sufficient and in most cases necessary to apply such constraints between the force constants that all the redundancy matrices $\tilde{\mathbf{F}}_{r}$ of eq. (22a) disappear, i.e., that all the vectors $\mathbf{h}_{r}$ become zero. We shall now see how this is accomplished in practice.

One way of constructing a uniquely defined force field in some redundant coordinates is to start with a set of nonredundant internal coordinates and make a transformation of the force constants into the redundant coordinate basis. The transformation is simple and requires no detailed knowledge about the 
redundancy relations, only the definitions of the nonredundant coordinates in terms of the redundant coordinates. This is easily seen by writing the potential energy in both bases. Thus, if the relation between a set of redundant coordiantes $q$ and a set of nonredundant coordinates $\mathbf{s}$ is

$$
\mathbf{s}=\mathbf{U q}
$$

the part of the potential energy that depends upon the force field can be written

$$
2 V=\Delta \mathbf{s}^{t} \mathbf{F}_{s} \Delta \mathbf{s}=\Delta \mathbf{q}^{t} \mathbf{U}^{t} \mathbf{F}_{s} \mathbf{U} \Delta \mathbf{q}=\Delta \mathbf{q}^{t} \mathbf{F} \Delta \mathbf{q}
$$

where $\mathbf{F}_{s}$ is the force constant matrix in the nonredundant coordinates and

$$
\mathbf{F}=\mathbf{U}^{t} \mathbf{F}_{s} \mathbf{U}
$$

is the force constant matrix in the redundant coordinates. This transformation automatically generates a valid choice of constraints in the force field. A simple example is the redundancy among the three valence angles in a planar trigonal system. If the angles are denoted $\alpha, \phi$, and $\phi^{\prime}$, a convenient nonredundant basis is formed by the angle $\alpha$ and the inplane wagging coordinate $\beta=1 / 2\left(\phi-\phi^{\prime}\right)$. (Another obvious choice would be the local symmetry coordinates for this group.) The force field to be transformed and the transformation matrix are then

$$
\mathbf{F}_{s}=\left(\begin{array}{ll}
F_{\alpha} & f_{\alpha \beta} \\
f_{\alpha \beta} & F_{\beta}
\end{array}\right) \text { and } \mathbf{U}=\left(\begin{array}{ccc}
1 & 0 & 0 \\
0 & \frac{1}{2} & -\frac{1}{2}
\end{array}\right)
$$

respectively, and the force field transformed into the redundant coordinate basis is

$$
\mathbf{F}=\left(\begin{array}{ccc}
F_{\alpha}^{\prime} & f_{\alpha \phi} & f_{\alpha \phi^{\prime}} \\
f_{\alpha \phi} & F_{\phi} & f_{\phi \phi^{\prime}} \\
f_{\alpha \phi^{\prime}} & f_{\phi \phi^{\prime}} & F_{\phi^{\prime}}
\end{array}\right)=\left(\begin{array}{ccc}
F_{\alpha} & \frac{1}{2} f_{\alpha \beta} & -\frac{1}{2} f_{\alpha \beta} \\
\frac{1}{2} f_{\alpha \beta} & \frac{1}{4} F_{\beta} & -\frac{1}{4} F_{\beta} \\
-\frac{1}{2} f_{\alpha \beta} & -\frac{1}{4} F_{\beta} & \frac{1}{4} F_{\beta}
\end{array}\right)
$$

When the force field is optimized, the constraints between the elements of $\mathbf{F}$ must be retained at all times; otherwise, the force field may become indeterminate. However, the choice of constraints is not unique, and many others than those shown in eq. (27) are equally well applicable. This has to be taken into account, e.g., when comparing different force fields. When a redundant basis is used, it is, of course, not correct to compare force constants that correspond to different choices of constraints, even if the coordinate basis is the same. Transformations between different representations of a force field given in a redundant basis are therefore sometimes necessary. To carry out such a transformation of a force field $\mathbf{F}$, each redundancy relation must be used to derive a matrix $\mathbf{F}_{r}$, as given by eq. (22a), and the new constraints must then be applied to the matrix

$$
\mathbf{F}^{\prime}=\mathbf{F}+\sum_{r} \tilde{\mathbf{F}}_{r}
$$

In the trigonal case, the redundancy relation is

$$
\Delta \alpha+\Delta \phi+\Delta \phi^{\prime}=0
$$

and the vectors $\mathbf{a}$ and $\mathbf{h}$ are given by $\mathbf{a}^{t}=(111)$ and $\mathbf{h}^{t}=\left(h_{1} h_{2} h_{3}\right)$, respectively, and the matrix $\tilde{\mathbf{F}}_{r}$ is

$$
\overline{\mathbf{F}}_{r}=\mathbf{a h}^{t}+\mathbf{h} \mathbf{a}^{t}=\left(\begin{array}{ccc}
2 h_{1} & h_{1}+h_{2} & h_{1}+h_{3} \\
h_{2}+h_{1} & 2 h_{2} & h_{2}+h_{3} \\
h_{3}+h_{1} & h_{3}+h_{2} & 2 h_{3}
\end{array}\right)
$$

By adding this matrix to that of eq. (27) and solving for the $h$ parameters when new constraints are applied, the force field can easily be transformed to correspond to any choice of constraints. For instance, to transform (27) into diagonal form we add (30) and set all the off-diagonal elements in the sum matrix equal to zero. The result is then

$$
\mathbf{F}=\left(\begin{array}{ccc}
F_{\alpha}-\frac{1}{4} F_{\beta} & 0 & 0 \\
0 & \frac{1}{2} F_{\beta}-f_{\alpha \beta} & 0 \\
0 & 0 & \frac{1}{2} F_{\beta}+f_{\alpha \beta}
\end{array}\right)
$$

If the molecular mechanics force field is optimized directly in a redundant coordinate basis, without any reference to a nonredundant basis, eq. (22a) can be used to check the validity of the constraints imposed on the force field. This is done by applying the same constraints to the elements of the matrix $\tilde{\mathbf{F}}_{r}$ and should yield $\mathbf{h}=0$. If a diagonal or almost diagonal force field is optimized, as has so far mostly been the case, the (implicit) constraints are normally strict enough to remove the indeterminacy. On the other hand, if one wants to construct a more accurate force field care should be taken to ensure that proper constraints are really used. It is easy to show that this is not trivial. In the trigonal case, at least three constraints (to fix the three $h$ parameters) are needed to remove the indeterminacy from the $\mathbf{F}$ matrix. However, any three constraints are not valid. For example, if we choose $F_{\phi}=F_{\phi^{\prime}}, f_{\alpha \phi}=f_{\alpha \phi^{\prime}}$, and $f_{\phi \phi^{\prime}}=0$, we get

$$
\begin{aligned}
2 h_{2} & =2 h_{3} \\
h_{1}+h_{2} & =h_{1}+h_{3} \\
h_{2}+h_{3} & =0
\end{aligned}
$$

which gives $h_{2}=h_{3}=0$ but does not fix $h_{1}$. Similarly, if we change the third constraint, $f_{\phi \phi^{\prime}}=0$, to $f_{\phi \phi^{\prime}}=$ $f_{\alpha \phi}$, the third row of (32) becomes $h_{2}+h_{3}=h_{1}+$ $h_{2}$ and the solution of eq. (32) is then $h_{1}=h_{2}=h_{3}$, which still contains one degree of freedom. These constraints therefore are not sufficient in an optimization of the force constants of a trigonal system. Improper constraints are indeed something to look out for because they may seem logical or reasonable but still be invalid.

A more complicated redundancy occurs among the six valence angles in a tetrahedral configuration, 
such as, for example, the methyl group attached to an atom $\mathrm{A}$. In this case, the redundancy relation is not linear, which means that the redundancy vector a depends upon the expansion center. Let us denote the $\mathrm{H}-\mathrm{C}-\mathrm{H}$ angles by $\alpha_{1}, \alpha_{2}$, and $\alpha_{3}$ and the A-C-H angles by $\beta_{1}, \beta_{2}$, and $\beta_{3}$. If perfect tetrahedral symmetry is assumed for $\mathbf{q}_{e x}=\mathbf{q}_{0}$, the cosine of each angle is $-1 / 3$ and the redundancy relation to the first order is

$$
\Delta \alpha_{1}+\Delta \alpha_{2}+\Delta \alpha_{3}+\Delta \beta_{1}+\Delta \beta_{2}+\Delta \beta_{3}=0
$$

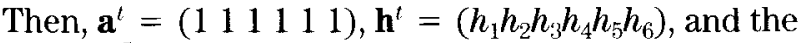
matrix $\tilde{\mathbf{F}}_{r}$ is constraints are sufficient. For example, if we require that $f\left(\alpha_{i}, \beta_{i}\right)=0, i=1,2,3$, and that $f\left(\alpha_{i}, \alpha_{j}\right)=f_{\alpha}$ and $f\left(\beta_{i}, \beta_{j}\right)=f_{b}$ (for $i \neq j$ ), only 14 force constants remain. But, these constraints are still insufficient because they do not fix the $h$ parameters, giving only that $h_{1}=h_{2}=h_{3}=h_{4}=h_{5}=h_{6}$. An additional constraint is needed to fix the $h$ parameters to zero, thus leaving only 13 independent force constants. As in the trigonal case, a transformation from a set of nonredundant coordinates can be used to circumvent the redundancy. This may often be preferable because it actually allows one to work with force

$$
\tilde{\mathbf{F}}_{r}=\left(\begin{array}{cccccc}
2 h_{1} & h_{1}+h_{2} & h_{1}+h_{3} & h_{1}+h_{4} & h_{1}+h_{5} & h_{1}+h_{6} \\
h_{2}+h_{1} & 2 h_{2} & h_{2}+h_{3} & h_{2}+h_{4} & h_{2}+h_{5} & h_{2}+h_{6} \\
h_{3}+h_{1} & h_{3}+h_{2} & 2 h_{3} & h_{3}+h_{4} & h_{3}+h_{5} & h_{3}+h_{6} \\
h_{4}+h_{1} & h_{4}+h_{2} & h_{4}+h_{3} & 2 h_{4} & h_{4}+h_{5} & h_{4}+h_{6} \\
h_{5}+h_{1} & h_{5}+h_{2} & h_{5}+h_{3} & h_{5}+h_{4} & 2 h_{5} & h_{5}+h_{6} \\
h_{6}+h_{1} & h_{6}+h_{2} & h_{6}+h_{3} & h_{6}+h_{4} & h_{6}+h_{5} & 2 h_{6}
\end{array}\right)
$$

If $\mathbf{q}_{e x}$ differs from perfect tetrahedral symmetry, the elements of a are not all 1 but instead $\left(1+\epsilon_{i}\right), i=$ $1, \ldots, 6$. The elements $h_{i}+h_{j}$ of $\tilde{\mathbf{F}}_{r}$ are then replaced by $\left(1+\epsilon_{j}\right) h_{i}+\left(1+\epsilon_{i}\right) h_{j}, i, j=1, \ldots, 6$. However, any set of constraints in $\tilde{\mathbf{F}}_{r}$ that fixes the $h$ parameters to zero for tetrahedral symmetry will do so also when the symmetry is broken. This is because all proper sets of linear constraints give rise to a homogeneous system of equations with respect to the $h$ parameters, and provided that the coefficient matrix does not become singular this always yields $\mathbf{h}=\mathbf{0}$. Thus, the same set of constraints removes the indeterminacy from the force field also when the expansion center, i.e., the intrinsic equilibrium geometry, does not correspond to perfect tetrahedral symmetry. At least six constraints are needed to eliminate the indeterminacy, leaving at most 15 independent parameters in the force constant matrix. A valid set of constraints is, for example, that the interaction force constants of type $f\left(\alpha_{i}, \beta_{j}\right)$ must be zero if $\alpha_{i}$ and $\beta_{j}$ do not share a bond and that $f\left(\alpha_{i}, \alpha_{j}\right)=f\left(\beta_{i}, \beta_{j}\right)$ for $i, j=1,2,3(i \neq j)$. Applied to the $\tilde{\mathbf{F}}_{r}$ matrix in (34), these constraints give lassuming that $\alpha_{i}$ and $\beta_{i},(i=1,2,3)$ do not share a bond]

$$
\begin{aligned}
& h_{1}+h_{4}=0 \\
& h_{2}+h_{5}=0 \\
& h_{3}+h_{6}=0
\end{aligned} \quad \text { and } \begin{aligned}
& h_{1}+h_{2}=h_{4}+h_{5} \\
& h_{1}+h_{3}=h_{4}+h_{6} \\
& h_{2}+h_{3}=h_{5}+h_{6}
\end{aligned}
$$

the only solution of which is $h_{i}=0, i=1, \ldots, 6$. These constraints leave the allowed maximum of 15 independent force constants. In actual optimization calculations, more strict constraints are generally preferable, although an exact transformation from, or comparison with, an ab initio or other complete force field is then not possible. However, even if the number of free parameters is smaller than the allowed maximum this does not guarantee that the constants pertaining to the nonredundant basis even if the basic molecular mechanics internal coordinates are redundant. The following local symmetry coordinates are a commonly used nonredundant basis for tetrahedral configurations:

$$
\begin{aligned}
& s_{1}=\frac{1}{\sqrt{6}}\left(\alpha_{1}+\alpha_{2}+\alpha_{3}-\beta_{1}-\beta_{2}-\beta_{3}\right) \\
& s_{2}=\frac{1}{\sqrt{6}}\left(2 \alpha_{1}-\alpha_{2}-\alpha_{3}\right) \\
& s_{3}=\frac{1}{\sqrt{2}}\left(\alpha_{2}-\alpha_{3}\right) \\
& s_{4}=\frac{1}{\sqrt{6}}\left(2 \beta_{1}-\beta_{2}-\beta_{3}\right) \\
& s_{5}=\frac{1}{\sqrt{2}}\left(\beta_{2}-\beta_{3}\right)
\end{aligned}
$$

Thus, if the force constant matrix in these coordinates has the diagonal elements $F_{i}, i=1, \ldots, 5$, and the off-diagonal elements $f_{i j}, i, j=1, \ldots, 5(i<j)$, application of transformation (25) gives the following for the force constants in the redundant coordinates:

$$
\begin{aligned}
F\left(\alpha_{1}, \alpha_{1}\right) & =\frac{1}{6} F_{1}+\frac{2}{3} F_{2}+\frac{2}{3} f_{12} \\
f\left(\alpha_{1}, \alpha_{2}\right)=\frac{1}{6} F_{1}-\frac{1}{3} F_{2}+\frac{1}{6} f_{12} & \\
& \quad+\frac{1}{\sqrt{12}} f_{13}+\frac{1}{\sqrt{3}} f_{23}
\end{aligned}
$$




$$
\begin{gathered}
f\left(\alpha_{1}, \alpha_{3}\right)=\frac{1}{6} F_{1}-\frac{1}{3} F_{2}+\frac{1}{6} f_{12} \\
\quad-\frac{1}{\sqrt{12}} f_{13}-\frac{1}{\sqrt{3}} f_{23} \\
f\left(\alpha_{1}, \beta_{1}\right)=-\frac{1}{6} F_{1}-\frac{1}{3} f_{12}+\frac{1}{3} f_{14}+\frac{2}{3} f_{24} \\
f\left(\alpha_{1}, \beta_{2}\right)=-\frac{1}{6} F_{1}-\frac{1}{3} f_{12}-\frac{1}{6} f_{14} \\
\quad+\frac{1}{\sqrt{12}} f_{15}-\frac{1}{3} f_{24}+\frac{1}{\sqrt{3}} f_{25} \\
\quad-\frac{1}{\sqrt{12}} f_{15}-\frac{1}{3} f_{24}-\frac{1}{\sqrt{3}} f_{25} \\
f\left(\alpha_{1}, \beta_{3}\right)=-\frac{1}{6} F_{1}-\frac{1}{3} f_{12}-\frac{1}{6} f_{14} \\
\quad+\frac{1}{3} f_{14}-\frac{1}{3} f_{24}+\frac{1}{\sqrt{3}} f_{34} \\
F\left(\alpha_{2}, \alpha_{2}\right)=\frac{1}{6} F_{1}+\frac{1}{6} F_{2}+\frac{1}{2} F_{3} \\
\quad-\frac{1}{3} f_{12}+\frac{1}{\sqrt{3}} f_{13}-\frac{1}{\sqrt{3}} f_{23} \\
f\left(\alpha_{2}, \alpha_{3}\right)=\frac{1}{6} F_{1}+\frac{1}{6} F_{2}-\frac{1}{2} F_{3}-\frac{1}{3} f_{12} \\
-\frac{1}{6} F_{1}+\frac{1}{6} f_{12}-\frac{1}{\sqrt{12}} f_{13} \\
f\left(\alpha_{1}\right)=
\end{gathered}
$$

$$
\begin{aligned}
& f\left(\alpha_{2}, \beta_{2}\right)=-\frac{1}{6} F_{1}+\frac{1}{6} f_{12}-\frac{1}{\sqrt{12}} f_{13}-\frac{1}{6} f_{14} \\
& +\frac{1}{\sqrt{12}} f_{15}+\frac{1}{6} f_{24}-\frac{1}{\sqrt{12}} f_{25}-\frac{1}{\sqrt{12}} f_{34}+\frac{1}{2} f_{35}
\end{aligned}
$$$$
f\left(\alpha_{2}, \beta_{3}\right)=-\frac{1}{6} F_{1}+\frac{1}{6} f_{12}-\frac{1}{\sqrt{12}} f_{13}-\frac{1}{6} f_{14}
$$$$
-\frac{1}{\sqrt{12}} f_{15}+\frac{1}{6} f_{24}+\frac{1}{\sqrt{12}} f_{25}-\frac{1}{\sqrt{12}} f_{34}-\frac{1}{2} f_{35}
$$

$$
\begin{aligned}
F\left(\alpha_{3}, \alpha_{3}\right)=\frac{1}{6} F_{1}+\frac{1}{6} F_{2} & +\frac{1}{2} F_{3} \\
& -\frac{1}{3} f_{12}-\frac{1}{\sqrt{3}} f_{13}+\frac{1}{\sqrt{3}} f_{23}
\end{aligned}
$$

$$
\begin{aligned}
f\left(\alpha_{3}, \beta_{1}\right)=-\frac{1}{6} F_{1}+\frac{1}{6} f_{12} & +\frac{1}{\sqrt{12}} f_{13} \\
& +\frac{1}{3} f_{14}-\frac{1}{3} f_{24}-\frac{1}{\sqrt{3}} f_{34}
\end{aligned}
$$

$$
\begin{aligned}
& f\left(\alpha_{3}, \beta_{2}\right)=-\frac{1}{6} F_{1}+\frac{1}{6} f_{12}+\frac{1}{\sqrt{12}} f_{13}-\frac{1}{6} f_{14} \\
& \quad+\frac{1}{\sqrt{12}} f_{15}+\frac{1}{6} f_{24}-\frac{1}{\sqrt{12}} f_{25}+\frac{1}{\sqrt{12}} f_{34}-\frac{1}{2} f_{35}
\end{aligned}
$$

$$
\begin{gathered}
f\left(\alpha_{3}, \beta_{3}\right)=-\frac{1}{6} F_{1}+\frac{1}{6} f_{12}+\frac{1}{\sqrt{12}} f_{13}-\frac{1}{6} f_{14} \\
-\frac{1}{\sqrt{12}} f_{15}+\frac{1}{6} f_{24}+\frac{1}{\sqrt{12}} f_{25}+\frac{1}{\sqrt{12}} f_{34}+\frac{1}{2} f_{35} \\
F\left(\beta_{1}, \beta_{1}\right)=\frac{1}{6} F_{1}+\frac{2}{3} F_{4}-\frac{2}{3} f_{14} \\
f\left(\beta_{1}, \beta_{2}\right)=\frac{1}{6} F_{1}-\frac{1}{3} F_{4}-\frac{1}{6} f_{14} \\
f\left(\beta_{1}, \beta_{3}\right)=\frac{1}{6} F_{1}-\frac{1}{3} F_{4}-\frac{1}{6} f_{14} \\
F\left(\beta_{2}, \beta_{2}\right)=\frac{1}{6} F_{1}+\frac{1}{6} F_{4}+\frac{1}{2} F_{5} \\
+\frac{1}{\sqrt{3}} f_{45}-\frac{1}{\sqrt{3}} f_{15}-\frac{1}{\sqrt{3}} f_{45} \\
f\left(\beta_{2}, \beta_{3}\right)=\frac{1}{\sqrt{3}} f_{45} \\
F\left(\beta_{3}, \beta_{3}\right)=\frac{1}{6} F_{1}+\frac{1}{6} F_{4}-\frac{1}{2} F_{5}+\frac{1}{3} f_{14} \\
+\frac{1}{2} F_{5}+\frac{1}{3} f_{14} \\
+\frac{1}{3} f_{15}+\frac{1}{\sqrt{3}} f_{45}
\end{gathered}
$$

These relations are appropriate to use if symmetry is utilized in the optimization of the force constants or if transformation from, or comparisons with, $a b$ initio force fields are made. Even if the linear combinations look somewhat complicated, they, or any others, are not difficult to use provided that transformation (25) has been automated. An automation algorithm that allows optimization of force constants pertaining to a nonredundant basis of form (23) must perform transformation (25) alphanumerically and keep track of the positions and weighting factors of the nonredundant force constants in the redundant F matrix. For instance, in the example above we can see that the nonredundant force constant $F_{1}$ of the local symmetry coordinate $s_{1}$ contributes to every element in the redundant $\mathbf{F}$ matrix, the weighting factor being either $+1 / 6$ or $-1 / 6$. Similarly, the force constant $F_{3}$ contributes to $F\left(\alpha_{1}, \alpha_{2}\right), f\left(\alpha_{2}, \alpha_{3}\right)$, and $F\left(\alpha_{3}, \alpha_{3}\right)$, weighted by $+1 / 2,-1 / 2$, and $+1 / 2$, respectively, and so on. With this information available, the nonredundant force constants can be optimized instead of the redundant ones, even though the redundant coordinate basis is retained.

\section{CONCLUSIONS}

As we have seen, there are two ways of dealing with the indeterminacy caused by redundancies. One can 
either work with force constants pertaining to a nonredundant basis by using eq. (25) or use a set of constraints between the force constants. The constraints must be such that, when applied to the matrix $\tilde{\mathbf{F}}_{r}$ of eq. (22a), this matrix vanishes. The latter alternative may seem simpler from a practical point of view but has a problem associated with it: The constraints need not correspond to physical reality. For example, in the trigonal and tetrahedral cases the requirement that all the diagonal force constants must be zero leads to a perfectly proper set of constraints. Clearly, though, such force constants do not reflect any physical properties of the internal coordinates involved. Even if less dramatic or seemingly physically reasonable constraints are used, the transferability of the force constants is not necessarily as good as that obtained with nonredundant coordinates. Therefore, to retain physically meaningful definitions of all energy parameters it may be worthwhile using force constants related to a nonredundant coordinate basis. The trigonal redundancy is especially simple and in this case it is certainly recommendable to use the in-plane wagging coordinate to avoid indeterminacy with respect to force constants within the trigonal group, as well as with respect to interaction force constants between the trigonal group and other coordinates. For tetrahedral configurations, the use of nonredundant coordinates is not as straightforward, although quite feasible, and it may well turn out that instead some simple constraints lead to physically reasonable and transferable force constants. Only experience will show whether or not this is the case. The same is true of cyclic redundancies in ring structures, an illustrative example of which has been given by Kydd in terms of a spectroscopic out-of-plane force field for benzene. ${ }^{11}$ In a procedure suggested by Halgren, ${ }^{12,13}$ a set of constraints could be combined with the requirement that the off-diagonal force constants be as small as possible. However, there is no obvious physical reason why the transferability of such force constants would be the best possible.

As regards torsion about a bond, several dihedral angles are usually associated with the bond in question. If each one of the dihedral angles is defined as a separate torsion coordinate, the corresponding barrier heights become intrinsically correlated due to the local redundancy. In spectroscopic force field calculations, the problem is easy to circumvent by using a single torsion coordinate defined as the mean of two or more of the dihedral angles. This is often a good enough approximation when calculating the vibrational frequencies at a known energy minimum. However, in molecular mechanics the tor- sion potentials are also expected to reproduce correct conformations and energy barriers, in addition to the frequencies, and one parameter per torsion may then not be sufficient. In such cases, the torsion parameters should be optimized to data from many different conformations, or determined by fitting to ad initio torsion potentials. For torsions about double bonds, though, the simple procedure used in spectroscopic force field calculations also works in molecular mechanics.114 In this case, the twofold two-component torsion coordinate introduced by Bell ${ }^{15}$ is probably the physically best motivated choice for the purpose of obtaining transferable force constants (barrier heights) both for the torsions and out-of-plane bending coordinates. The force constants in the redundant (many torsions per bond) basis can, as before, be computed using eq. (25). Alternatively, explicit redundancy relations can also be obtained for the dihedral angles defined about the same bond. For example, as shown by Schlick, ${ }^{16}$ if $\tau_{1}$ and $\tau_{2}$ are the dihedral angles defined, respectively, for the bonded atom sequences $\{i, j, k$, $\left.l_{1}\right\}$ and $\left\{i, j, k, l_{2}\right\}$, and $\theta_{1}, \theta_{2}, \theta_{3}$ are, respectively, bond angles $\left\{j, k, l_{1}\right\},\left\{j, k, l_{2}\right\}$, and $\left\{l_{1}, k, l_{2}\right\}$, then $\tau_{1}$ and $\tau_{2}$ are related by

$$
\cos \left(\tau_{1}-\tau_{2}\right)=\frac{\cos \theta_{3}-\cos \theta_{1} \cos \theta_{2}}{\sin \theta_{1} \sin \theta_{2}} .
$$

This research was supported by The Program in Protein Structure and Design at the University of Michigan, by a grant from the Monsanto Company, St. Louis, MO, and by NSF Grants DMB-8816756 and DMR-8806975.

\section{References}

1. L.-O. Pietilä, J. Mol. Struct, 195, 111 (1989).

2. K. Palmö, L.-O. Pietilä, and S. Krimm, J. Comp. Chem., 12, 385 (1991).

3. E.B. Wilson, Jr., J.C. Decius, and P.C. Cross, Molecular Vibrations, McGraw-Hill, New York, 1955.

4. B. Crawford, Jr. and J. Overend, J. Mol. Spectrosc., 12, 307 (1964).

5. S.J. Cyvin, Acta Chem. Scand., 20, 2616 (1966).

6. I.M. Mills, Chem. Phys. Lett., 3, 267 (1969).

7. P. Groner and H.H. Günthard, J. Mol. Spectrosc., 61, 151 (1976).

8. R. Meyer, P. Groner, and H.H. Günthard, Chem. Phys., 19, 407 (1977).

9. K. Kuczera, J. Mol. Struct., 160, 159 (1987).

10. S. Califano, Vibrational States, Wiley, New York, 1976.

11. R.A. Kydd, Spectrochim. Acta, 27A, 2067 (1971).

12. T.A. Halgren, J. Mol. Struct. (Theochem), 163, 431 (1988).

13. T.A. Halgren, J. Am. Chem. Soc., 112, 4710 (1990).

14. B. Mannfors, L.-O. Pietilä, and K. Palmö, J. Mol. Struct., 248, 289 (1991).

15. R.P. Bell, Trans. Faraday Soc., 41, 293 (1945).

16. T. Schlick, J. Comp. Chem., 9, 861 (1988). 